\title{
COMMENT
}

\section{The RESOLUTE consortium: unlocking SLC transporters for drug discovery}

\author{
Giulio Superti-Furga ${ }^{1,2 凶}$, Daniel Lackner, ${ }^{1,4}$, Tabea Wiedmer ${ }^{1}$, Alvaro Ingles-Prieto', \\ the RESOLUTE consortium* and Claire M. Steppan ${ }^{3 凶}$
}

The Innovative Medicines Initiative consortium RESOLUTE has started to develop tools and produce data sets to de-orphanize transporters in the solute carrier protein (SLC) superfamily, thereby lowering the barrier for the scientific community to explore SLCs as an attractive drug target class.

The solute carrier proteins (SLCs) comprise a superfamily of transporters controlling the import and export of molecules across membranes. With more than 450 members, SLCs are the largest family of transporters encoded by the human genome. Although some members are successful drug targets, such as glucose and neurotransmitter transporters, the vast majority of SLCs have not been exploited therapeutically. In fact, many SLCs are 'orphans' in terms of knowledge of their substrate specificity and biological function.

To unlock the therapeutic potential of this superfamily, the RESOLUTE consortium - a public-private partnership funded by the European Innovative Medicines Initiative (IMI) and in-kind contributions from the European Federation of Pharmaceutical Industries and Associations (EFPIA) - has initiated a systematic programme to generate tools for the scientific community to tackle this target class, while providing data aiming at de-orphanizing members on a large scale. Here, we outline the rationale for this programme, describe the progress so far and the next steps, and call for community action to help achieve the consortium goals.

CeMM Research Center for Molecular Medicine of the Austrian Academy of Sciences, Vienna, Austria.

${ }^{2}$ Center for Physiology and Pharmacology, Medical University of Vienna, Vienna, Austria.

${ }^{3}$ Pfizer Worldwide Research, Development and Medical, Groton, USA.

${ }^{4}$ Deceased: Daniel Lackner.

*The entire list of consortium members and authors can be found in the online version of the Comment.

凶e-mail:gsuperti@

cemm.oeaw.ac.at;

Claire.M.Steppan@pfizer.com

https://doi.org/10.1038/

d41573-020-00056-6

\section{The necessity for coordinated action on SLCs}

The relevance of SLCs for drug discovery is increasingly being acknowledged ${ }^{1}$. Recent studies are providing mechanistic insights into SLC function, and these studies are also going beyond traditional approaches for studying transporters, such as injection of mRNA in Xenopus laevis oocytes, by applying technologies from the field of genomics and systems biology, such as genome-wide CRISPR-interference screens (for example, see REFS' ${ }^{2,3}$ ). There are also recent examples in which new modulators of SLCs have been identified, such as chromopynones that target glucose transporters ${ }^{4}$ and SLC39A7 inhibitors identified in phenotypic screen $s^{5}$.

Nevertheless, most SLCs have been difficult to access, owing to a lack of appropriate research tools, a lack of generic assays, a lack of appreciation of their redundant and integrated roles and a lack of chemical probes. This largely untapped source of new potential drug targets therefore merits a systematic and coordinated approach to study the entire family, rather than focusing single, separate efforts on individual SLCs.

The RESOLUTE consortium has been established to address this challenge, with the primary initial objective of creating a comprehensive set of long-lasting, well-characterized tools and data packages to enable research on the SLC family. RESOLUTE consists of 13 partners: 6 academic institutions (CeMM Research Center for Molecular Medicine of the Austrian Academy of Sciences, Leiden University, Max Planck Institute for Medical Research, University of Liverpool, University of Oxford and the University of Vienna), 6 pharma companies (Pfizer, Bayer, Boehringer Ingelheim, Novartis, Sanofi and Vifor Pharma) and 1 small or medium-sized enterprise (Axxam SpA).

The ultimate goal for the consortium - large-scale de-orphanization of the SLC superfamily - can be likened to matchmaking. On one hand, there are a finite number of SLC transporters, and on the other, a finite number of metabolites from various origins (endogenous, bacterial, environmental and food). We expect that matchmaking between transporters and metabolites will be difficult at the beginning until a tipping point, following which exclusion principles can be applied. So, large-scale de-orphanization of SLCs can only be achieved if the scientific community is sufficiently engaged in matching each SLC to a set of transported molecules and a cellular function. Every contribution matters, hence the plea associated with this article.

\section{RESOLUTE's research plan}

The research plan of the consortium is divided into two complementary approaches. In the first approach, tools, reagents and data will be generated for as many SLCs as possible, independently of predicted function, relevance to medical research or the degree to which they have been studied. In the second, parallel approach, the consortium will focus more on a subset of 'priority targets', 
particularly functional assay development and the generation of protein reagents. This priority set will be based on criteria including medical interest, orphan status and data generated by the consortium.

RESOLUTE is following an open-access ethos, and so data and reagents are being made publicly available via the RESOLUTE web portal and public repositories. The web portal contains two main resources: the RESOLUTE public database (see Related links), which provides access to data produced and released by the consortium, and the RESOLUTE knowledgebase (see Related links), which compiles data from multiple public sources.

The consortium has started with the generation of reliable and validated tools and data sets. Owing to the complex biochemical properties of integral membrane proteins, SLCs often show low expression when transfected in mammalian cells. We recently generated 443 DNA sequences of SLCs codon-optimized for expression in human cells and inserted them in a vector compatible with the Gateway recombination cloning technology. RESOLUTE's plasmid collection is available in Addgene (see Related links). More DNA reagents will be added to the collection during 2020.

We have selected six adherent human cancer cell lines (HCT-116, Huh7, LS180, MDA-MB-468, SK-MEL-28 and $1321 \mathrm{~N} 1$ ) that cumulatively cover the expression of approximately $80 \%$ of all SLCs (considering a cut-off of $\geq 1$ transcripts per million), based on a publicly available RNA-sequencing data set. Additionally, we selected HEK293 JumpIn T-REx to generate clonal-like and stable overexpressing control cell lines. We have characterized the parental cell lines and released data on the transcriptome, proteome and abundance of major metabolites, which are accessible through the RESOLUTE public database.

The next step is creating knockout and tagged overexpression cell lines for the majority of SLCs, which are at the core of the de-orphanization process. Our initial hope is to be able to de-orphanize SLCs using a 'guilt by association' strategy that leverages omics (transcriptomics, metabolomics, proteomics and ionomics) data generated by comparing isogenic SLC-knockout cells with SLC-overexpression cells. De-orphanization and subsequent demonstration of functionality for an orphan SLC will be challenging, but we are only at the beginning of the process, so we cannot yet assess our success or the full extent of the challenge. To date, we have generated 440 SLC-overexpressing cell lines, 100 SLC-knockout cell lines and 20 cell lines in which SLCs are overexpressed on a knockout background. In late 2020 we will start depositing cell lines in a public cell repository and expect a continuous release until reaching an estimated total number of 350 SLC-knockout, 350 SLC-knockout-overexpressing and 350 HEK293 SLC-overexpressing cells by 2023 . The results from omics analyses and functional assays will be released as data sets and in scientific publications from 2020 onwards.

For selected SLCs - mainly the priority targets we are generating high-affinity protein binders using various approaches, including monoclonal antibodies, nanobodies and sybodies. Following assessment of specificity and affinity employing our SLC cell lines, we will publish the sequences of binders starting in 2021. We are also assessing the suitability of SLCs for a particular assay technology systematically, taking phylogenetic and other relationships into account. Such an 'assay-finding engine' is conceived to efficiently advance as many SLCs as possible through a pipeline of different experimental set-ups. For a selected number of SLCs, these assays will be further developed to generate cellular and/or cell-free assay systems that are suitable for high-throughput screening campaigns.

Finally, a data analysis pipeline is being developed that will allow efficient exploitation of the vast amount of data generated. The data generated by RESOLUTE are being combined with existing data from the literature and public databases to form the foundation of an SLC-focused database, which is gradually becoming accessible to the scientific community upon data release from the consortium to the public domain. Integration of complex data sets is another challenge to be tackled.

\section{SLCs to enter the target class canon}

RESOLUTE's ambition is to contribute in a decisive manner to the addition of SLC transporters to the canon of classic target classes: G protein-coupled receptors, kinases, proteases and ion channels. This addition is long overdue, given that the potential number of SLCs exceeds 450 , their proven tractability as drug targets (including for blockbuster drug classes such as selective serotonin re-uptake inhibitors targeting SLC6A4 for depression and glifozins targeting SLC5A2 for type 2 diabetes), their involvement in a wide range of diseases and the growing appreciation of the role of metabolism in pathophysiology. This article is intended to act as a communication of the work in progress and to motivate the community to access the tools, reagents and protocols developed by the consortium to generate data and compounds that unlock the therapeutic potential of SLC transporters.

\footnotetext{
1. Lin, L., Yee, S. W., Kim, R. B. \& Giacomini, K. M. SLC transporters as therapeutic targets: emerging opportunities. Nat Rev Drug. Discov. 14, 543-560 (2015)

2. Luteijn, R. D. et al. SLC19A1 transports immunoreactive cyclic dinucleotides. Nature 573, 434-438 (2019).

3. Girardi, E. et al. A widespread role for SLC transmembrane transporters in resistance to cytotoxic drugs. Nat. Chem. Biol. https://doi.org/10.1038/s41589-020-0483-3 (2020).

4. Karageorgis, G. et al. Chromopynones are pseudo natural product glucose uptake inhibitors targeting glucose transporters GLUT-1 and -3. Nat. Chem 10, 1103-1111 (2018)

5. Nolin, E. et al. Discovery of a ZIP7 inhibitor from a Notch pathway screen. Nat. Chem. Biol. 15, 179-188 (2019).
}

\section{Acknowledgements}

This project has received funding from the Innovative Medicines Initiative 2 Joint Undertaking under grant agreement No. 777372 . This Joint Undertaking receives support from the European Union's Horizon 2020 research and innovation programme and EFPIA.

\section{Competing interests}

Although research of the RESOLUTE consortium is in the precompetitive space, the following authors are employed by companies as indicated in more detail by their affiliations: M.C., R.E., D.H., S.L., S.N., M.P., R.S., C.M.S., H.Z., S.C., A.F., M.I., G.M., L.R., F.S., L.S., M.S., P.T., S.T., H.B., A.B., E.B., Y.N.C. A.E., A.M.F. V.P., D.Z., B.H., M.L., D.S., C.V., C.W., B.B., L.C., Y.I., M.P., J.R., J.S., P.S., K.S., C.B., B.B., J.C., M.D., H.F., T.L., V.M., A.P., F.P., V.Y., P.A., C.D., F.D., V.M., A.L.S., H.S., M.W.

\section{RELATED LINKS}

RESOLUTE database: https://re-solute.eu/database

RESOLUTE knowledgebase: https://re-solute.eu/knowledgebase RESOLUTE's plasmid collection: https://www.addgene.org/browse/ article/28206712 